\title{
Influência do Tipo de Amostragem na Estimativa de Volume de Madeira de Eucalipto por Krigagem
}

\author{
Wellington Jorge Cavalcanti Lundgren ${ }^{1}$, José Antônio Aleixo da Silva², \\ Rinaldo Luiz Caraciolo Ferreira ${ }^{2}$ \\ ${ }^{1}$ Departamento Unidade Acadêmica de Serra Talhada, Universidade Federal Rural de Pernambuco - UFRPE, \\ Serra Talhada/PE, Brasil \\ ${ }^{2}$ Departamento de Ciência Florestal, Universidade Federal Rural de Pernambuco - UFRPE, Recife/PE, Brasil
}

\begin{abstract}
RESUMO
O objetivo da pesquisa foi verificar se a estimativa do volume individual de madeira por árvore realizada pela krigagem é influenciada pelo tipo de amostragem. Um povoamento com 1.875 árvores de eucalipto foi demarcado em um plano cartesiano $(\mathrm{X}, \mathrm{Y})$ e cubado rigorosamente pelo método de Smalian. Foram retiradas seis amostragens, cada uma contendo 106 árvores. Duas amostragens por gradeamento, duas aleatórias e duas por grade aglomerada. Os semivariogramas para cada uma das amostragens e mapas do volume individual das árvores foram construídos por krigagem. Onze tipos de erros foram comparados e ranqueados. A amostragem aleatória apresentou os melhores resultados e a amostragem grade aglomerada, os piores.
\end{abstract}

Palavras-chave: volume de madeira, semivariograma, geoestatística, dependência espacial, gradeamento.

\section{Sampling Type Influence in the Estimation of Eucalyptus Wood Volume by Kriging}

\begin{abstract}
The objective was to verify if the estimate of the individual volume of wood per tree conducted by kriging is influenced by the type of sampling. A settlement with 1875 eucalyptus trees was marked on a Cartesian plane (X, Y) and strictly cubed using the Smalian method. Six samples were taken, each containing 106 trees. Two samples per railing, two random samples and two by crowded grid. The semivariograms were constructed for each of the sampling and maps of the individual volume of trees were constructed by kriging. Eleven types of errors were compared and ranked. Random sampling showed the best results and clustered sampling grid showed the worst results.
\end{abstract}

Keywords: wood volume, semivariogram, geostatistics, spatial dependence, railing. 


\section{INTRODUÇÃO}

Um dos fatores que eleva o custo do inventário florestal é o tamanho da amostra. A dificuldade surge pela exigência de que ela seja a menor possível sem perder, contudo, a precisão do resultado. Vários autores realizaram pesquisas sobre o método e o número de amostras utilizados em inventários florestais (Cesaro et al., 1994; Vibrans et al., 2010; Leite \& Andrade, 2002).

O procedimento usual é a regressão da estatística clássica, que leva em conta a hipótese de que as amostras são independentes entre si. Hipótese que nem sempre corresponde à verdade. Diniz (2007) afirma que se o atributo não tem dependência espacial, então a estatística clássica é utilizada, fornecendo o menor número de amostras necessário. Porém, se a dependência espacial existir, métodos que levem em conta essa característica fornecem resultados precisos utilizando menor número de amostras do que a estatística clássica.

A geoestatística se apresenta como ferramenta que vem auxiliar as ciências florestais, diminuindo o custo do inventário e fornecendo resultados tão confiáveis quanto os conseguidos pela estatística clássica, alguns autores já a utilizaram e conseguiram resultados precisos (Mello et al., 2006; Lundgren et al., 2015; Santana, 2011).

Outra vantagem da geoestatística é a não necessidade de voltar a campo para realização de medições para efetuar estimação, por exemplo, do volume de madeira. Com a geoestatística é possível construir um mapa que fornece a estimativa do volume baseando-se apenas na localização da árvore no campo.

Na geoestatística, a amostragem usual é a formação de uma grade de linhas no campo em estudo e a retirada das amostras nos cruzamentos entre as linhas.

Pode-se ainda retirar as amostras aleatoriamente dentro do campo. Esse processo é pouco usual, geralmente é utilizado quando os pontos amostrais não podem ser escolhidos e já fazem parte da estrutura de pesquisa como, por exemplo, em estudos sobre pluviosidade em que as estações de chuva já existem e não podem ser mudadas de local apenas para se realizar a pesquisa (Cavalcanti \& Corrêa, 2014; Montebeller et al., 2007).

$\mathrm{O}$ distanciamento entre os pontos amostrados deve ser pequeno o bastante para que a dependência espacial seja detectada. No entanto, se os pontos forem muito próximos, o número de amostras será elevado, fato que aumenta o custo do inventário.

O objetivo da atual pesquisa é verificar se existe diferença entre três métodos de amostragem para os resultados da krigagem do volume individual de madeira de eucalipto.

\section{MATERIAL E MÉTODOS}

A pesquisa foi realizada na Serra do Araripe, localizada no município de Araripina, PE, nas coordenadas geográficas $7^{\circ} 27^{\prime} 37^{\prime \prime} \mathrm{S}$ e $40^{\circ} 24^{\prime} 36^{\prime \prime} \mathrm{W}$ e na altitude de 831 metros (Barros, 2009).

Um povoamento de eucaliptos com 1.875 árvores em uma área de $178 \times 134$ metros $=2,39$ ha com 6 anos de idade e com espaçamento $2 \times 3 \mathrm{~m}$ contendo 15 diferentes tipos de clones (ver Tabela 1) plantados em parcelas de $7 \times 7$ árvores e divididos em 4 blocos, separados entre si por uma estrada de 6 metros de largura foi todo cubado rigorosamente pelo método de Smalian.

As árvores foram demarcadas em um plano cartesiano $(\mathrm{X}, \mathrm{Y})$ onde $\mathrm{X}$ e $\mathrm{Y}$ são a distância em metros da projeção dos pontos nos eixos para a árvore origem $(0,0)$, a qual pode ser visualizada na Figura 1.

Foram utilizados três tipos de amostragens diferentes e cada uma delas foi repetida duas vezes (A e B). A repetição teve por objetivo a tentativa de confirmar a superioridade de um método sobre o outro. Cada amostragem continha 106 amostras, as árvores existentes nos vértices do povoamento nas posições $(0,0),(0,138),(174,0)$ e $(174,138)$ foram incluídas nas amostras impositivamente, para fechar o polígono, permitindo a construção do mapa do volume por krigagem.

Os tipos de amostragem foram:

a) Amostra inteiramente casualizada;

b) Gradeamento;

c) Aglomerado.

As amostragens $a$ e $b$ são procedimentos usuais, não necessitando de maiores explicações, o procedimento $c$, chamado aqui de Aglomerado, consistiu em se retirar amostras no nó das grades, com espaçamentos homogêneos entre os nós e mais duas árvores próximas a eles formando um L. A distribuição das amostras 
Tabela 1. Clones de Eucalyptus sp. usados no Módulo de Experimentação Florestal para a região do Araripe, em Araripina, PE.

Table 1. Clones from Eucalyptus sp. used in Forest Experiment Module for the Region of the Araripe in Araripina, PE.

\begin{tabular}{|c|c|c|}
\hline Tratamento & Clone & Descrição \\
\hline 01 & $\mathrm{C} 49$ & E. tereticornis (cruzamento natural) \\
\hline 02 & $\mathrm{C} 80$ & Híbrido de E. urophylla x E. tereticornis (polinização controlada) \\
\hline 03 & C315 & Híbrido de E. urophylla x E. tereticornis (polinização controlada) \\
\hline 04 & $\mathrm{C} 101$ & Híbrido de E. urophylla x E. tereticornis (polinização controlada) \\
\hline 05 & $\mathrm{C} 78$ & Híbrido de E. urophylla x E. tereticornis (polinização controlada) \\
\hline 06 & C156 & Híbrido de E. urophylla x E. tereticornis $\times$ E. pllita (polinização controlada) \\
\hline 07 & C39 & E. urophylla (polinização controlada) \\
\hline 08 & $\mathrm{C} 27$ & E. brassiana (cruzamento natural) \\
\hline 09 & C51 & Híbrido de E. urophylla x E. tereticornis (polinização controlada) \\
\hline 10 & $\mathrm{C} 158$ & Híbrido de E. urophylla x E. tereticornis (polinização controlada) \\
\hline 11 & $\mathrm{C} 41$ & E. urophylla (cruzamento natural) \\
\hline 12 & C31 & E. brassiana (cruzameno natural) \\
\hline 13 & $\mathrm{C} 25$ & E. brassiana (cruzamento natural) \\
\hline 14 & $\mathrm{C} 33$ & E. urophylla (cruzamento natural) \\
\hline 15 & $\mathrm{C} 11$ & E. brassiana (cruzamento natural) \\
\hline
\end{tabular}

retiradas e a posição das parcelas com os seus clones estão apresentadas na Figura 1.

Não foi possível retirar as amostras em grade de forma totalmente padronizada, com espaçamentos rigorosamente iguais, devido às falhas existentes no povoamento, porém é possível perceber que estão organizadas de forma a se aproximar de um gradeamento. $\mathrm{O}$ mesmo pode ser afirmado para as amostras aglomeradas.

As estatísticas básicas, média, mediana, moda, máximo, mínimo, desvio padrão, curtose, assimetria e o teste de normalidade Kolmogorov-Smirnov foram realizados pelos softwares GS+ e Minitab 16 . A transformação logarítmica foi utilizada para tentar normalizar os dados.

Em geoestatística, segundo Landim et al. (2002), trabalha-se com o pressuposto de que a média e a covariância espacial são homogêneas em determinada área. Para a verificação desses pressupostos realiza-se a análise de tendência, que foi verificada conforme o procedimento descrito por Gonçalves et al. (2001), no qual gráficos de dispersão são construídos com os valores do eixo $\mathrm{X} \times$ Volume e o eixo $\mathrm{Y} \times$ Volume: caso exista correlação de moderada a forte entre os valores fica comprovada a existência de tendência.

Para a retirada da tendência, uma equação de regressão é ajustada para o gráfico de dispersão e um semivariograma é construído com os resíduos (diferença entre os valores observados e os valores estimados pela equação).

A principal ferramenta da geoestatística, segundo Landim (2006), é o semivariograma, um gráfico de dispersão que correlaciona a distância entre os pontos amostrais com a semivariância. A semivariância amostral é calculada pela Fórmula 1 .

$\mathrm{Y}(\mathrm{h})=\frac{1}{2 . N(h)} \sum_{\mathrm{i}=1}^{\mathrm{N}(\mathrm{h})}\left[\mathrm{Z}_{\mathrm{i}}-\mathrm{Z}_{\mathrm{i}+\mathrm{h}}\right]^{2}$

em que: $\mathrm{Y}(\mathrm{h})$ = semivariância amostral; $\mathrm{N}(\mathrm{h})$ = número de pares de pontos que se encontram separados pela distância $\mathrm{h} ; \mathrm{h}=$ distância entre dois pontos amostrais; $\mathrm{i}$ = ponto de localização da amostra [par ordenado $(\mathrm{x}, \mathrm{y})] ; \mathrm{i}+\mathrm{h}=$ todos os pontos distantes do ponto $\mathrm{i}$ até no máximo à distância $\mathrm{h} ; \mathrm{Z}_{\mathrm{i}}=$ valores do atributo no ponto $\mathrm{i} ; \mathrm{Z}_{\mathrm{i}+\mathrm{h}}=$ valor do atributo no ponto com distância h do ponto i.

O semivariograma teórico trabalha essencialmente com três parâmetros que são utilizados na modelagem dos dados:

O alcance, que é a distância máxima até onde se percebe o efeito da dependência espacial;

- O patamar, que é semivariância encontrada exatamente onde está o alcance; 

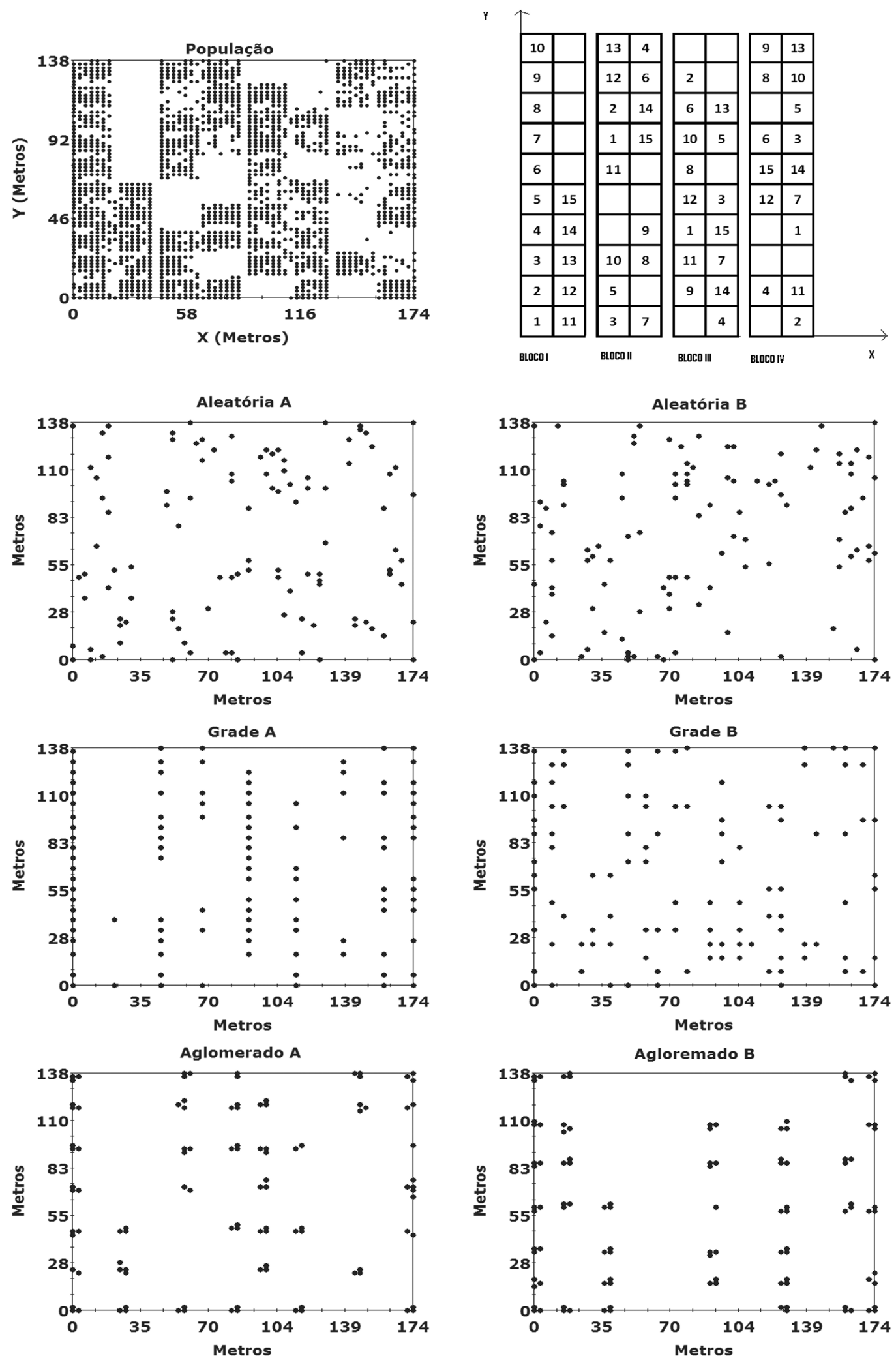

Figura 1. Posição das árvores no campo, distribuição das parcelas com os clones e posição das amostras retiradas. Figure 1. Position of the trees in the field, distribution of plots with the clones and position of the samples taken. 
- O efeito pepita, que é a semivariância encontrada quando a distância é zero. O efeito pepita representa as microvariações não captadas pelas amostras.

Existem alguns modelos teóricos que comumente são utilizados para a modelagem do semivariograma amostral - os mais utilizados são os Modelos 2-5:

$$
\begin{aligned}
& Y(h)=C_{0}+\frac{h C}{A} \text { Linear } \\
& Y(h)=C_{0}+C\left[1,5\left(\frac{h}{A}\right)-0,5\left(\frac{h}{A}\right)^{3}\right] \text { Esférico } \\
& Y(h)=C_{0}+C\left[1-\operatorname{Exp}\left(\frac{-h}{A}\right)\right] \text { Exponencial } \\
& Y(h)=C_{0}+C\left[1-\operatorname{Exp}\left(\frac{-3 h}{A}\right)^{2}\right] \text { Gaussiano }
\end{aligned}
$$

em que: $\mathrm{C}_{0}=$ efeito pepita; $\mathrm{C}=$ patamar; $\mathrm{A}=$ alcance; $\mathrm{e} \mathrm{h}=$ distância.

A anisotropia é uma possível característica do atributo. Ocorre quando o atributo apresenta diferentes comportamentos espaciais para diferentes direções. Para verificar a existência de anisotropia, Landim et al. (2002) recomenda a construção de semivariogramas nas direções $0^{\circ}, 45^{\circ}, 90^{\circ}$ e $135^{\circ}$. Quando os semivariogramas apresentam patamar, alcance e efeito pepita diferentes para alguma das direções, a anisotropia fica comprovada. E um novo semivariograma é ajustado para caracterizar a anisotropia.

Para a quantificação do Índice de Dependência Espacial (IDE) foi utilizada a relação IDE = efeito pepita/patamar se IDE $>0,75$, dependência fraca, $0,25 \leq \mathrm{IDE} \leq 0,75$, dependência moderada, e se IDE $<0,25$, dependência forte.

Escolhido o modelo matemático para o semivariograma teórico, foi usada a krigagem simples para a construção dos mapas de volume. A krigagem é calculada pela Fórmula 6.

$$
Z_{k}=\sum_{i=1}^{N} \lambda_{i} Z_{i+h}
$$

Com a exigência de que a Equação 7 exista.

$$
\sum_{i=1}^{N} \lambda_{i}=1
$$

em que: $Z_{k}=$ valor estimado do atributo no ponto k; $\mathrm{Z}_{\mathrm{i}+\mathrm{h}}=$ valor do atributo com distância máxima $\mathrm{h}$ do ponto $\mathrm{k}$ a ser estimado; $\lambda_{\mathrm{i}}=$ peso do atributo.

Os critérios para a escolha do melhor delineamento amostral incluem vários tipos de erros, usando-se a seguinte equação: $\mathrm{E}_{\mathrm{i}}=\mathrm{VV}_{\mathrm{i}}-\mathrm{VE}_{\mathrm{i}}$, em que $\mathrm{E}_{\mathrm{i}}$ é o erro da estimação do volume da árvore i; $\mathrm{VV}_{\mathrm{i}}=$ volume verdadeiro da árvore $\mathrm{i} ; \mathrm{VE}_{\mathrm{i}}=$ volume estimado da árvore i.

Histogramas dos erros $\mathrm{E}_{\mathrm{i}}$ foram construídos para cada uma das amostragens. Os erros devem apresentar distribuição normal com média zero e variância a menor possível.

Foram atribuídos escores de 0 (zero) a 6 para cada amostragem, as menores notas para os melhores resultados, a soma dos escores com menor valor caracterizou o melhor tipo de amostragem.

Os erros utilizados foram:

1) Erro médio quadrático (EQM). Equação 8.

$E Q M=\sum_{i=1}^{N} \frac{E_{i}^{2}}{N}$

em que: $\mathrm{N}$ = número total de árvores.

2) Erro da estimação total do volume do povoamento (ETV). Equação 9.

$\mathrm{ETV}=\mathrm{VE}-\mathrm{VV}$.

em que: $\mathrm{VE}=$ volume total estimado do povoamento; $\mathrm{VV}=$ volume verdadeiro do povoamento.

3) Desvio padrão dos erros (DPE). Equação 10.

$D P E=\sqrt{\frac{1}{n-1}\left[\sum_{i=1}^{n} E_{i}^{2}-\frac{\sum_{i=1}^{n} E_{i}}{n}\right]}$

em que: $\mathrm{n}=$ número de amostras.

4) Assimetria dos erros (ASE). Equação 11.

$A S E=\frac{3(\text { Média }- \text { Mediana })}{D P E}$

5) Curtose dos erros (CUE). Equação 12.

$C U E=\frac{\text { Terceiroquartil }- \text { Primeiro quartil }}{2(\text { Percentil } 90-\text { Percentil } 10)}$ 
6) Desvio médio dos erros. (DME). Equação 13

$D M E=\sum_{i=1}^{N} \frac{\left|E_{i}\right|}{N}$

7) Erro percentual médio. (EPM). Equação 14.

$E P M=\sum_{i=1}^{N} \frac{\left(E_{i}\right)}{V V_{i}} \cdot 100$

Equação de regressão linear simples. Equação 15.

$V V_{i}=\alpha \cdot V E_{i}+\beta$

8) Gráfico de dispersão. Equação 16.

$\mathrm{VE}_{\mathrm{i}} \times \mathrm{VV}_{\mathrm{i}}$

9) $\mathrm{R}^{2}$ do gráfico de dispersão. Equação 17.

$\mathrm{VE}_{\mathrm{i}} \times \mathrm{VV}_{\mathrm{i}}$

10) Coeficiente angular da regressão linear simples para o gráfico de dispersão (CAR). Equação 18.

$\mathrm{VE}_{\mathrm{i}} \times \mathrm{VV}_{\mathrm{i}}$

11) Intercepto da regressão linear simples para o gráfico de dispersão (IR). Equação 19.

$\mathrm{VE}_{\mathrm{i}} \times \mathrm{VV}_{\mathrm{i}}$

\section{RESULTADOS E DISCUSSÃO}

Na Tabela 2 podem ser visualizadas as estatísticas básicas de cada uma das seis amostras, além dos dados populacionais.
Tomando como base os dados populacionais, nota-se que em geral as medidas de posição foram superestimadas em todas as amostras, excetuando-se a mediana estimada pela aleatória $\mathrm{A}$. O desvio padrão e o CV foram estimados bem próximos dos valores reais. A curtose e o Máximo foram subestimados pelas amostras e o mínimo e a assimetria foram superestimados, exceto pela assimetria da aleatória A.

O teste de normalidade Kolmogorov-Smirnov rejeitou a distribuição normal para todas as amostras e para a população. A transformação logarítmica foi realizada e a normalidade dos dados foi conseguida.

A análise de tendência foi realizada para cada uma das amostras.

A tendência foi encontrada nas seguintes amostragens:

1) No eixo $Y$ na amostra em grade $A$, o modelo para ajuste foi o quadrático.

2) Nos eixos $X$ e $Y$ na amostra em grade $B$, com ajuste quadrático em ambos os eixos.

3) Nos eixos $\mathrm{X}$ e $\mathrm{Y}$ na amostra aleatória $\mathrm{A}$, os modelos para ajuste foram lineares para ambos os eixos.

4) Nos eixos $\mathrm{X}$ e $\mathrm{Y}$ nas amostras aglomeradas A e B, os modelos de ajuste foram quadráticos em ambos os casos.

5) Não foi encontrada tendência na amostra aleatória B.

Tendências foram encontradas por Gonçalves et al. (2001) em estudo sobre a granulometria do solo no município de Piracicaba, SP. Fizeram uma análise

Tabela 2. Estatísticas básicas do volume $\left(\mathrm{cm}^{3}\right)$. Est = estatísticas; Pop = população; Aleat = aleatória; Aglo=aglomerada; Méd = média; $\mathrm{Md}=$ mediana; $\mathrm{Mo}=$ moda; Desv = desvio padrão; Cur = curtose; Ass = assimetria; Máx = máximo; Mín = mínimo; $\mathrm{CV}=$ coeficiente de variação.

Table 2. Basic statistics to volume $\left(\mathrm{cm}^{3}\right)$. Est = statistics; Pop = population; Aleat = random; Aglo = crowded; Méd = average; Md = median; Mo = mode; Desv = standard deviation; Cur = kurtosis; Ass = asymmetry; Máx = Maximum; Mín = Minimum; $C V=$ coefficient of variation .

\begin{tabular}{cccccccc}
\hline Est & Pop & Grade A & Grade B & Aleat A & Aleat B & Aglo A & Aglo B \\
\hline Méd & 88.807 & 113.366 & 97.185 & 93.162 & 99.865 & 109.312 & 118.811 \\
Md & 74.990 & 99.931 & 76.579 & 72.190 & 85.059 & 85.772 & 99.059 \\
\hline Mo & 55.251 & 99.914 & 62.999 & 75.615 & 92.935 & 72.760 & 68.841 \\
\hline Desv & 52.330 & 64.793 & 59.276 & 60.632 & 58.703 & 68.809 & 65.357 \\
\hline Cur & 6,59 & 1,36 & 2,65 & 6,37 & 3,78 & 2,49 & 1,16 \\
\hline Ass & 2,07 & 1,15 & 1,54 & 2,28 & 1,79 & 1,5 & 1,15 \\
\hline Máx & 467.665 & 348.558 & 348.558 & 376.659 & 348.558 & 376.659 & 348.558 \\
\hline Mín & 7.047 & 17.032 & 12.606 & 22.490 & 7.727 & 19.131 & 19.479 \\
\hline CV & 58,93 & 57,15 & 60,99 & 65,08 & 58,78 & 62,95 & 55,01 \\
\hline
\end{tabular}


aprofundada das implicações da existência de tendências nos resultados obtidos pela geoestatística e utilizaram o método do ajuste de polinômio pelo processo dos mínimos quadrados.

Para a construção dos mapas pela krigagem é necessário que sejam construídos os semivariogramas amostrais e teóricos, os dois estão apresentados juntos, com os respectivos mapas de krigagem, na Figura 2. Nenhum dos seis semivariogramas apresentou efeito pepita puro, permitindo a construção dos mapas e mostrando que a dependência espacial existe.

Visualmente, os mapas apresentaram estruturas bem diferenciadas umas das outras, provocando a incerteza de qual dos seis mapas forneceu as melhores estimativas volumétricas.

Mapas de volume de madeira de eucalipto também foram construídos por Carvalho et al. (2012). Eles construíram os mapas usando krigagem e cokrigagem e compararam os resultados. Afirmam que a krigagem forneceu melhor resultado que a cokrigagem. Para a comparação, utilizaram a validação cruzada.

Os valores dos parâmetros dos semivariogramas estão apresentados na Tabela 3.

O alcance é um parâmetro particularmente importante, pois auxilia outros pesquisadores do mesmo atributo a decidir o espaçamento que irão utilizar entre as amostras. Mello (2004) afirma que quanto maior o alcance, mais homogênea é a área, o que ajuda a fornecer melhores estimativas para a geoestatística, ao mesmo tempo que demanda um menor número de amostras.

O menor alcance na atual pesquisa foi do modelo esférico, para a amostragem aglomerada B, 9,80 metros, e o maior da aleatória $B, 38,40$ metros.
É importante frisar que as unidades experimentais foram volumes de árvores individuais e não parcelas. Em pesquisa em que a unidade foi volume por parcela, o alcance encontrado por Leal et al. (2014) foi de 1.100 metros.

Em todos os três tipos de amostragem, os valores dos alcances para as repetições A e B não forneceram valores próximos um do outro, ou seja, o tipo de amostragem não influenciou no valor do alcance.

Os efeitos pepitas foram próximos nas amostragens aleatórias A e B e nas amostragens aglomeradas A e B, com forte diferença para as amostragens em grade $A$ e $B$. Os parâmetros patamar foram próximos para as três amostragens.

Para os três tipos de amostragem não houve coincidência no modelo teórico das repetições (A e B). As amostras em grade apresentaram moderada dependência espacial

Os histogramas dos erros individuais da krigagem para cada uma das repetições das amostragens são apresentados na Figura 3. Todos os histogramas apresentaram a forma característica da distribuição normal; a distribuição dos erros ser normal é um indício de boas estimativas.

A amostragem aglomerada B apresentou em sua distribuição de erros a moda negativa e uma ligeira assimetria à direita, contendo a maioria dos erros negativos, indicando que as estimativas foram tendenciosas, ou seja, existiram mais estimativas a maior do verdadeiro valor.

Os erros das estimativas para cada uma das amostragens e suas repetições foram calculados e são apresentados na Tabela 4 . O total de volume do povoamento era de $166,14 \mathrm{~m}^{3}$, para o ETV, a amostra

Tabela 3. Parâmetros dos semivariogramas das amostragens. Gra = amostragem em grade; Ale = amostragem aleatória; $\mathrm{Agl}=$ amostragem aglomerada; $\mathrm{C}_{0}=$ efeito pepita; $\mathrm{P}=$ patamar; $\mathrm{A}=$ alcance; Exp = exponencial; Esf = esférico; Gau = gaussiano.

Table 3. Parameter semivariograms of sampling. Gra = grid sampling; Ale = random sampling; Agl = clustered sampling; $\mathrm{C}_{0}=$ nugget effect; $\mathrm{P}=$ sill; $\mathrm{A}=$ ranger; $\mathrm{Exp}=$ exponential; Esf = spherical; $\mathrm{Gau}=\mathrm{Gaussian}$.

\begin{tabular}{ccccccc}
\multirow{2}{*}{ Parâmetros Amostras } \\
\cline { 2 - 7 } & Gra A & Gra B & Ale A & Ale B & Agl A & Agl B \\
C $_{0}$ & 0,1793 & 0,0010 & 0,1102 & 0,1252 & 0,0769 & 0,0008 \\
P & 0,3596 & 0,3250 & 0,2614 & 0,3064 & 0,3558 & 0,2836 \\
A & 22,00 & 15,60 & 19,00 & 38,40 & 23,50 & 9,80 \\
Modelo & Exp. & Esf. & Gau. & Esf. & Exp. & Esf. \\
IDE & 0,4986 & 0,0031 & 0,4216 & 0,4086 & 0,2161 & 0,0023 \\
\hline
\end{tabular}



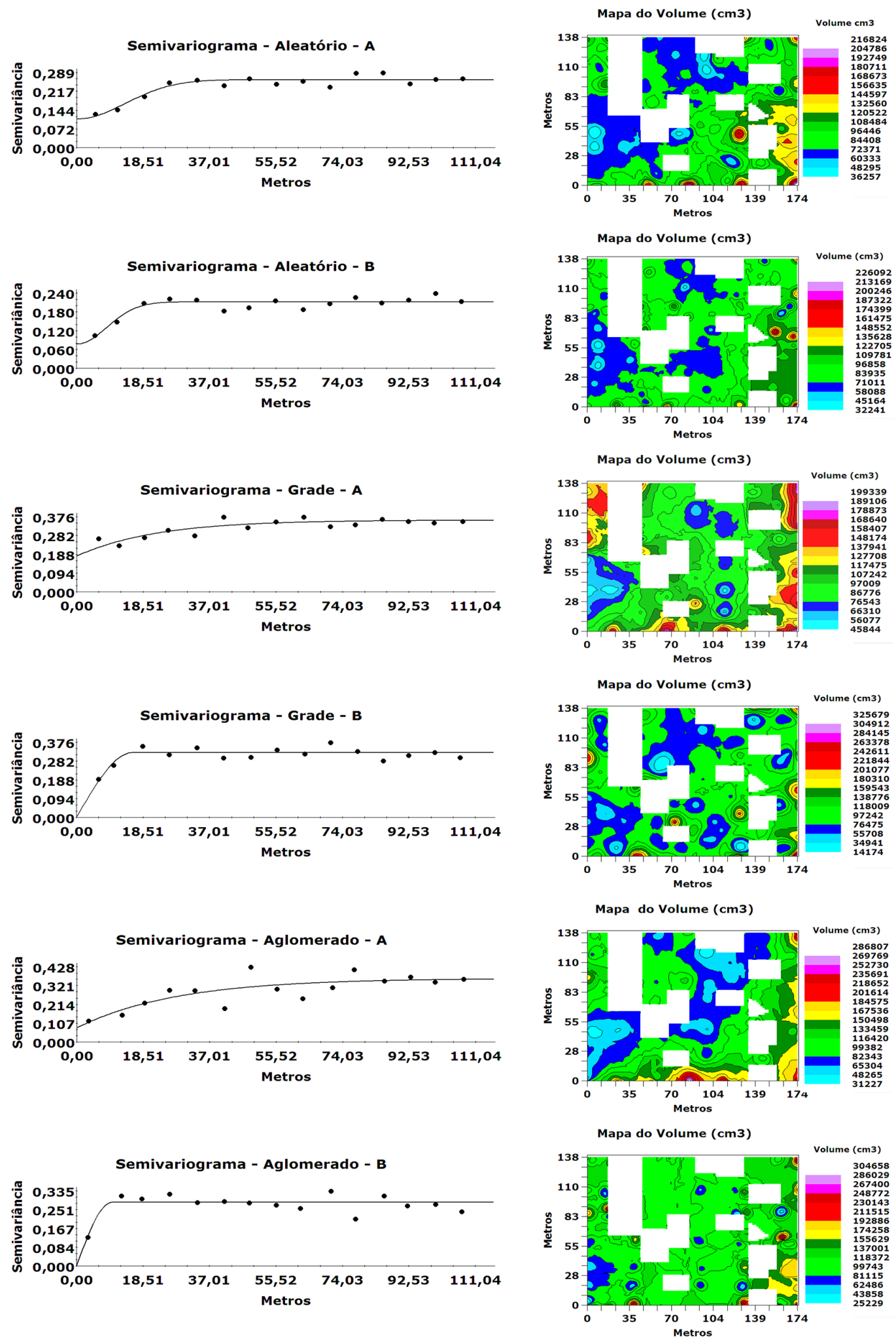

Figura 2. Semivariogramas e respectivos mapas de volume de madeira construídos por krigagem para seis amostragens diferentes.

Figure 2. Semivariograms and their wood volume maps constructed by kriging for six different samples. 
Histograma dos erros da Grade A

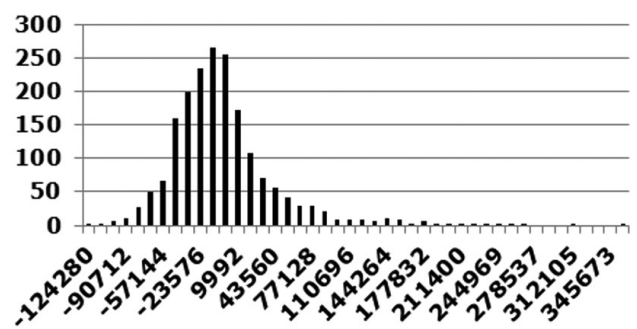

Histograma dos erros da Aleatória $\mathrm{A}$

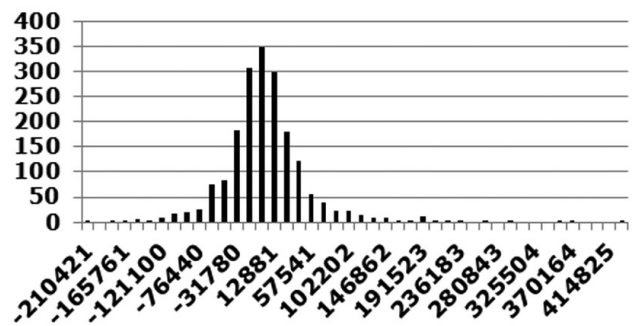

Histograma dos erros da Aglomerada A

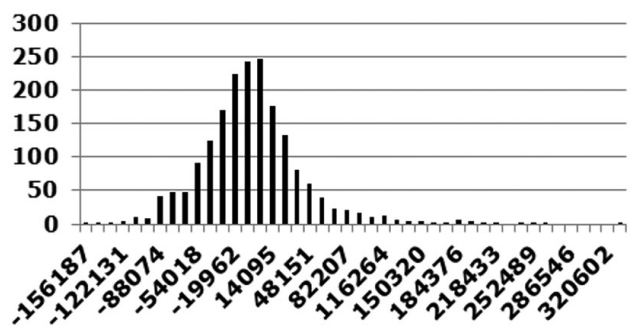

Histograma dos erros da Grade B

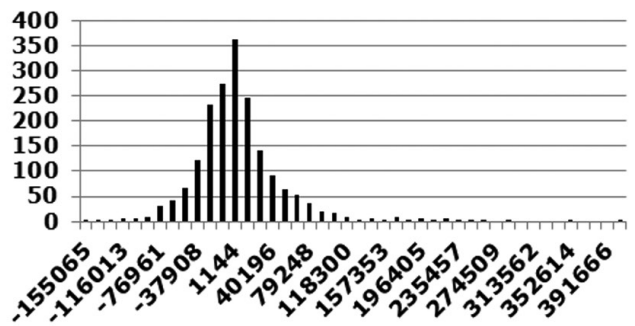

Histograma dos erros da Aleatória B

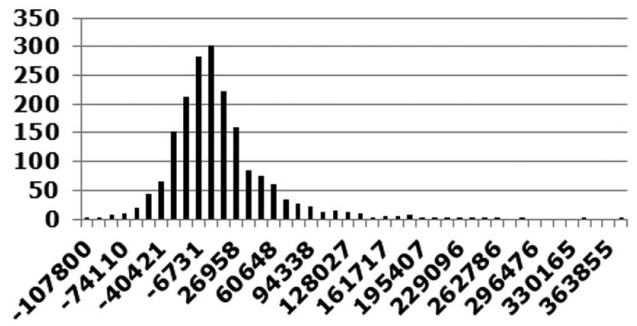

Histograma dos erros da Aglomerada B

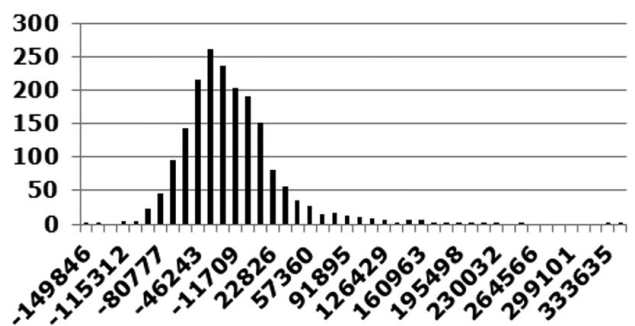

Figura 3. Histogramas dos erros individuais da krigagem para cada uma das amostragens.

Figure 3. Histograms of individual errors kriging for each of the samples.

Tabela 4. Erros das amostragens e suas repetições. $\mathrm{E}=$ escore; $\mathrm{A}$ e B = repetições das amostragens; ETV = erro total do volume; EMQ = erro média quadrático; DPE = desvio padrão dos erros; ASE = assimetria dos erros; $\mathrm{CUE}=$ curtose dos erros; $\mathrm{DME}=$ desvio médio dos erros; $\mathrm{EPM}=$ erro percentual médio; $\mathrm{CAR}=$ coeficiente angular da regressão; IR = intercepto da regressão.

Table 4. Errors of sampling and its repetitions. $\mathrm{E}=\mathrm{drain}$; $\mathrm{A}$ and $\mathrm{B}=$ repetitions of sampling; ETV = total volume error; EMQ = Error quadratic average; DPE = errors Standard deviation; ASE = Asymmetry of errors; CUE = kurtosis of the errors; $\mathrm{DME}=$ average deviation of the errors; $\mathrm{EPM}=$ mean percentage error; CAR $=$ angular coefficient of regression; IR = regression intercept.

\begin{tabular}{|c|c|c|c|c|c|c|c|c|c|c|c|c|}
\hline \multirow{3}{*}{ Erros } & \multicolumn{12}{|c|}{ Tipos de amostragem } \\
\hline & \multicolumn{4}{|c|}{ Grade } & \multicolumn{4}{|c|}{ Aleatória } & \multicolumn{4}{|c|}{ Aglomerada } \\
\hline & $\mathbf{A}$ & $\mathbf{E}$ & B & $\mathbf{E}$ & $\mathbf{A}$ & $\mathbf{E}$ & B & $\mathbf{E}$ & $\mathbf{A}$ & $\mathbf{E}$ & B & $\mathbf{E}$ \\
\hline ETV & $-17,45$ & 3 & $-1,60$ & 0 & $-7,22$ & 1 & 11,19 & 2 & $-18,2$ & 4 & $-38,79$ & 5 \\
\hline EMQ & 2.378 & 2 & 2.334 & 1 & 2.597 & 4 & 2.153 & 0 & 2.393 & 3 & 2.630 & 5 \\
\hline DPE & 47.921 & 2 & 48.310 & 4 & 50.819 & 5 & 46.016 & 0 & 47.956 & 3 & 46.933 & 1 \\
\hline ASE & 0,4567 & 3 & 0,3150 & 2 & 0,2159 & 1 & 0,4769 & 5 & 0,1588 & 0 & 0,4640 & 4 \\
\hline CUE & 0,3758 & 5 & 0,2115 & 0 & 0,2188 & 1 & 0,2244 & 2 & 0,2283 & 3 & 0,2449 & 4 \\
\hline DME & 35.615 & 4 & 32.242 & 1 & 34.393 & 2 & 30.616 & 0 & 35.529 & 3 & 39.955 & 5 \\
\hline EPM & 41,09 & 3 & 26,38 & 1 & 31,47 & 2 & 19,04 & 0 & 41,44 & 4 & 59,27 & 5 \\
\hline $\mathrm{R}^{2}$ & 0,163 & 1 & 0,178 & 2 & 0,135 & 5 & 0,228 & 0 & 0,193 & 3 & 0,195 & 4 \\
\hline CAR & 0,901 & 2 & 0,708 & 3 & 0,567 & 5 & 1,099 & 1 & 0,707 & 4 & 0,968 & 0 \\
\hline IR & 673,35 & 0 & 25.314 & 4 & 36.160 & 5 & -2.230 & 1 & 19.151 & 3 & -17.166 & 2 \\
\hline Total & & 25 & & 23 & & 27 & & 11 & & 30 & & 35 \\
\hline
\end{tabular}


grade $\mathrm{B}$ forneceu resultado quase exato, com diferença de $-1,60 \mathrm{~m}^{3}$, ou seja, erro percentual de $-0,9 \%$, porém a grade A forneceu $-17,45 \mathrm{~m}^{3}$, erro percentual de $-10,5 \%$, estimativas muito diferentes entre si.

Rocha et al. (2010) compararam estimativas de volume de madeira de eucalipto por 11 métodos diferentes com o volume real de 459 árvores de um povoamento em Vitória da Conquista, BA. Dos 11 métodos, quatro forneceram erros menores que 5\%, e Leite \& Andrade (2002) estimaram volume total de um plantio de eucalipto usando o método da altura relativa e conseguiram um erro de $12,16 \%$, considerando que os resultados foram satisfatórios.

A amostragem aglomerada também forneceu resultados destoantes para ETV, diferença de mais que o dobro entre os erros, que são os maiores entre as amostragens. Os melhores resultados para o ETV foram para a amostragem aleatória.

Os EMQ foram muito semelhantes entre todas as amostragens, sendo a melhor estimativa da aleatória B e a pior, a da aglomerada B, o EMQ foi utilizado por Conceição (2004) quando comparou três métodos de estimativa de volume de madeira de eucalipto no município de Agudos, SP. Os métodos comparados foram o método de Smalian, fator de forma e função de afilamento: ele chegou à conclusão de que não existe diferença significativa entre os métodos.

Para os valores do DPE, a aleatória B forneceu o melhor resultado e a aleatória $\mathrm{A}$, o pior entre todas as amostras. Para o DPE, todos os valores das amostragens foram próximos.

Nos histogramas da Figura 3, todos foram aparentemente simétricos em relação à moda. No cálculo da ASE, quanto mais perto de 0 (zero), mais simétrica será a distribuição. Todos os resultados indicaram assimetria positiva e o melhor resultado foi o da aglomerada $\mathrm{A}$; o pior, o da aleatória $\mathrm{B}$.

Os erros apresentaram distribuição Normal, portanto, tinham simetria quase perfeita e média próxima a 0 (zero), padrão da qualidade das estimativas. Porém, na natureza esse não é um fato concreto. Apesar da importância dessa verificação, poucos trabalhos verificam a simetria dos erros de seus dados. Lima (2012) descreve a importância da verificação da simetria dos erros para estimativas realizadas por regressão, porém não verifica a simetria dos erros.
Quanto à curtose da distribuição dos erros (CUE), o melhor resultado foi o da grade A e o pior, o da grade B. Excetuando-se o resultado da grade B, todos os outros resultados forneceram distribuição leptocúrtica, indicando que as estimativas nesse aspecto foram boas, pois o que se deseja é que a média dos erros seja próxima a 0 (zero) e que a dispersão seja pequena e esteja acumulada em torno dessa média.

O DME forneceu o melhor resultado com o aleatório $\mathrm{B}$ e o pior com o aglomerado B. O desvio médio absoluto foi utilizado por Imaña-Encinas et al. (2009) em comparação de equações volumétricas para estimar o volume total de madeira em área localizada no cerrado, na região de Planaltina de Goiás, fronteira com o Distrito Federal.

O EPM teve o melhor resultado com a aleatória B e o pior com a aglomerada B. Couto et al. (1989) utilizaram o EPM na comparação de dois modelos de regressão para volume de madeira de eucalipto que utilizavam como variáveis explicativas índice de sítio, idade da árvore e altura dominante. Os modelos foram testados em dois ciclos, talhadia e alto fuste, eles obtiveram EPM bem menores do que os observados na pesquisa aqui apresentada: o modelo 1 para a talhadia apresentou $\mathrm{EPM}=0,55 \%$ e para o alto fuste, $\mathrm{EPM}=14,25 \%$; o modelo 2 para a talhadia obteve $\mathrm{EPM}=-3,65 \%$ e, para o alto fuste, $\mathrm{EPM}=-0,68$.

Na Figura 4 estão apresentados os gráficos de dispersão dos volumes Estimados x Observados, juntamente com os valores dos $\mathrm{R}^{2} \mathrm{e}$ a respectiva equação de regressão linear simples $Y=a X+b$, em que: $Y=$ volume verdadeiro da árvore, $X=$ volume estimado da árvore, $a$ e $b$ são os coeficientes da equação.

O melhor $\mathrm{R}^{2}$ dos erros foi o da aleatória $\mathrm{B}$ e o pior, da aleatória $\mathrm{A}$; mesmo assim os valores foram muito abaixo do que pode ser considerado um bom resultado de previsão, já que $\mathrm{R}^{2}$ acima de 0,70 é indício de boa estimativa, porém vale ressaltar que foram usados 1.875 pontos, o que dificulta o ajustamento da reta, já que o $\mathrm{CV}$ dos dados foi próximo de $59 \%$, variação não considerada baixa.

Thaines et al. (2010) compararam 7 modelos de equação de regressão para a estimativa de volume de madeira de 141 árvores utilizando 4 tipos de comparadores: $\mathrm{R}^{2}$, erro padrão da estimativa, coeficiente de variação e valor de F na análise de variância, eles escolheram 
Estimados x Observados - Grade A

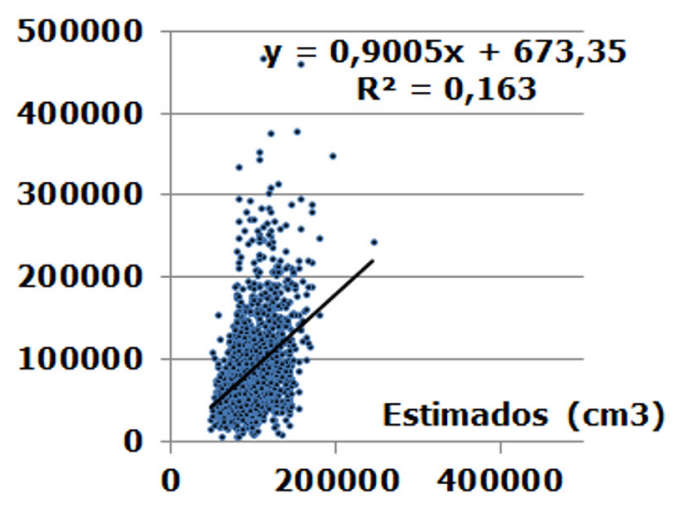

\section{Estimados x Observados - Aleatória A}

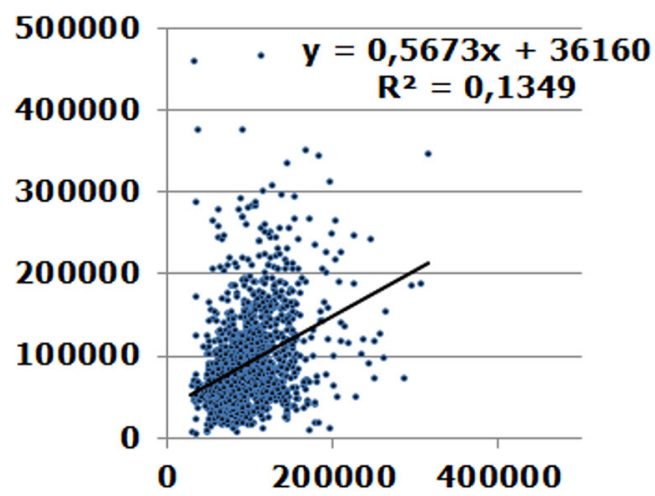

Estimados x Observados - Aglomerado A

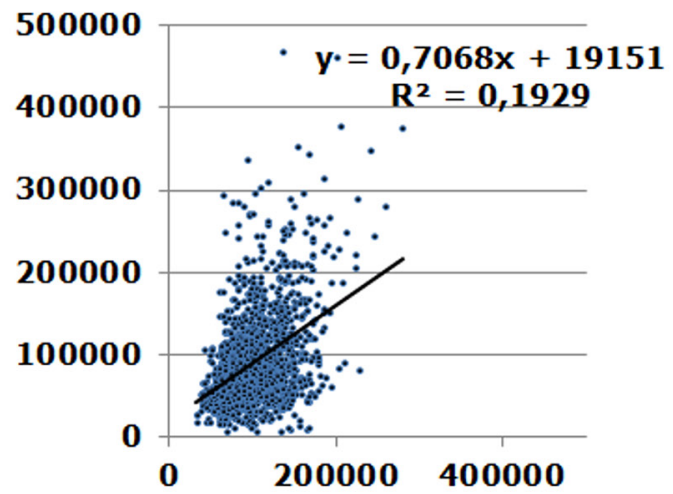

Estimados x Observados - Grade B

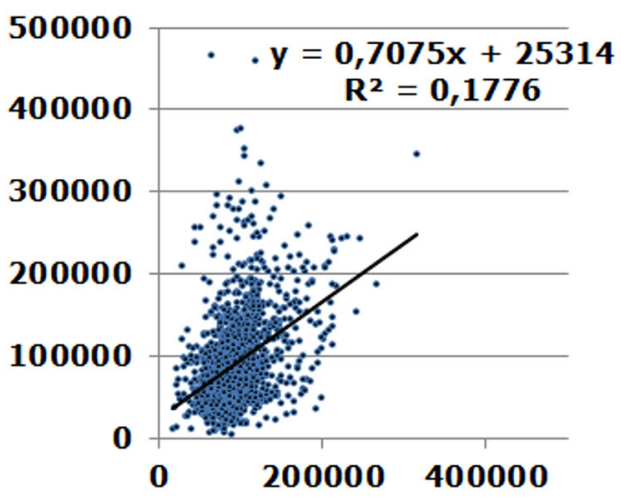

Estimados x Observados - Aleatória B

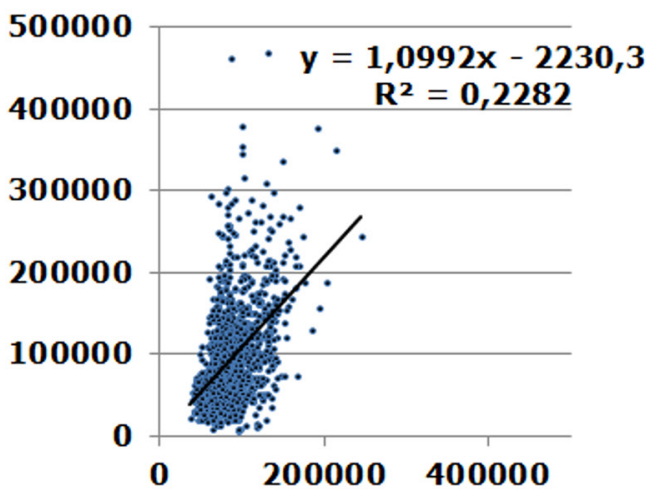

Estimados x Observados - Aglomerado B

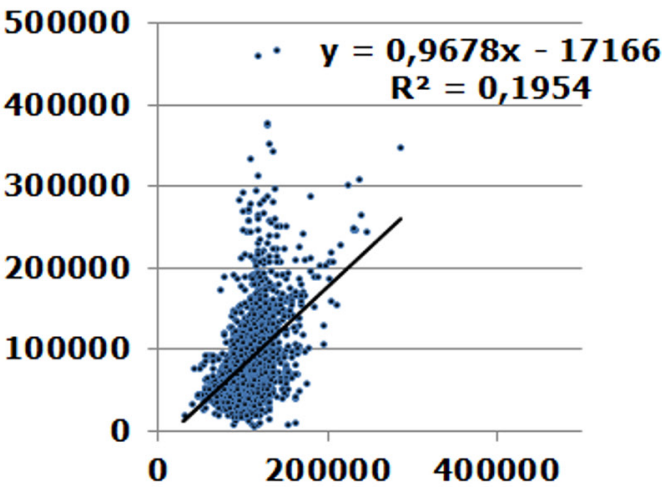

Figura 4. Gráficos de dispersão de Valores estimados $\times$ Valores observados.

Figure 4. Scatter plots of estimated $\times$ values observed values.

o melhor modelo por escore. Eles obtiveram valores de $\mathrm{R}^{2}$ altos, maiores que 0,82 .

O melhor CAR foi o da aglomerada B e o pio, o da aleatória $\mathrm{A}$, os valores da aleatória $\mathrm{B}$ e aglomerado $B$ não estão distantes de 1 , o que seria o ideal. Leite \& Andrade (2002) comentam sobre a importância do coeficiente angular para equações que estimam o volume de madeira: eles conseguiram CARs muito próximos a 1 em equações de regressão que utilizaram como variáveis explicativas o DAP e a altura da árvore.

Os valores dos interceptos (IR) foram melhores na grade $\mathrm{A}$, seguidos pelos da aleatória $\mathrm{B}$, e os piores foram da aleatória A. Como os dados estão $\mathrm{em}^{\mathrm{cm}^{3}}$, a grandeza dos números é na casa de cinco dígitos, o 
que torna os resultados dos erros na grade A $(673,35)$, bem próximos a 0 (zero). No trabalho de Leite \& Andrade (2002), os IRs foram melhores do que os aqui apresentados; o pior IR que eles conseguiram foi para o modelo que usava a altura relativa, e mesmo assim o valor do intercepto dos volume Estimados $\mathrm{x}$ Observados foi próximo à 0 (zero).

\section{CONCLUSÃO}

As estimativas realizadas por krigagem são influenciadas pelo tipo de amostragem utilizada.

A melhor amostragem foi a aleatória.

O tipo de erro escolhido para comparar estimadores de volume de madeira pode influenciar nas conclusões.

\section{AGRADECIMENTOS}

Os autores agradecem ao IPA - Instituto Agronômico de Pernambuco e ao CNPq - Conselho Nacional de Desenvolvimento Científico e Tecnológico.

\section{STATUS DA SUBMISSÃO}

Recebido: 7 mar., 2015

Aceito: 2 nov., 2015

\section{AUTOR(ES) PARA CORRESPONDÊNCIA}

\section{Wellington Jorge Cavalcanti Lundgren}

Departamento Unidade Acadêmica de Serra Talhada, Universidade Federal Rural de Pernambuco - UFRPE

Campus avançado, Fazenda saco, s/n, CEP 56912-194, Serra Talhada, PE, Brasil e-mail: wellingtonlundgren@yahoo.com.br.

\section{REFERENNCIAS}

Barros BC. Volumetria, densidade, carbono e calorimetria de espécies nativas e exóticas no pólo gesseiro do Araripe - PE [dissertação]. Recife: Departamento de Ciências Florestais, Universidade Federal Rural de Pernambuco; 2009.

Carvalho MP, Mendonça, VZ, Pereira FCBL, Arf MV, Kappes C, Dalchiavon, FC. Produtividade de madeira do eucalipto correlacionada com atributos do solo visando ao mapeamento de zonas específicas de manejo. Revista Ciência Rural 2012; 42(10): 1707-1803.
Cavalcanti LCS, Corrêa ACB. Pluviosidade no parque nacional do Catimbau (Pernambuco): seus condicionantes e seus efeitos sobre a paisagem. Geografia 2014; 23(2): 133-156.

Cesaro A, Engel AO, Finger CAG, Schneider PR. Comparação dos métodos de amostragem de área fixa, relascopia, e de seis árvores, quanto a eficiência, no inventário florestal de um povoamento de Pinus sp. Ciência Florestal 1994; 4(1): 97-108.

Conceição MB. Comparação de métodos de estimativa de volume em diferentes idades em plantações de Eucalyptus grandis Hill ex - Maiden [dissertação]. Curitiba: Setor de Ciências Agrarias, Universidade Federal do Paraná; 2004.

Couto HTZ, Bastos NLM, Lacerda JS. Comparação de dois modelos de prognose de madeira de Eucalyptus para alto fuste e talhadia. IPEF 1989; 41/42: 27-35.

Diniz FS. Métodos de amostragem e estudo aplicados ao inventário florestal [dissertação]. Lavras: Área de concentração em florestas de produção, Universidade Federal de Lavras; 2007.

Gonçalves ACA, Folegatti MV, Mata J DV. Análises exploratória e geoestatística da variabilidade de propriedades físicas de um Argissolo Vermelho. Revista Acta Scientiarum 2001; 23(5): 1149-1157

Imaña-Encinas J, Santana AO, Paula JE, Imaña CR. Equações de volume de madeira para o cerrado de Planaltina de Goiás. Floresta 2009; 39(1): 107-116. http:// dx.doi.org/10.5380/rf.v39i1.13731.

Landim PMB, Sturaro JR, Monteiro RC. Exemplos de aplicação da cokrigagem [online]. Rio Claro: UNESP; 2002 [citado em 2015 set 10]. Disponível em: http:// www.rc.unesp.br/igce/aplicada/DIDATICOS/LANDIM/ cokrigagem.pdf.

Landim PMB. Sobre geoestatística e mapas. Terra e Didática 2006; 2(1): 19-33.

Leal FA, Matricardi EAT, Miguel EP. Interpolador geoestatístico para estimar volume num povoamento de Eucalyptus urophylla, em Rio Verde/Goiás. Nucleus 2014; 11(1): 153-159. http://dx.doi.org/10.3738/1982.2278.1021.

Leite HG, Andrade VCL. Um método para condução de inventários florestais sem o uso de equações volumétricas. Revista Árvore 2002; 26(3): 321-328. http://dx.doi. org/10.1590/S0100-67622002000300007.

Lima LMA Fo. Modelos simétricos não lineares de produção e crescimento em volume de clones de Eucalyptus ssp [tese]. Recife: Departamento de Ciências Florestais, Universidade Federal Rural de Pernambuco; 2012.

Lundgren WJC, Silva JAA, Ferreira RLC. Estimação do volume de madeira de eucalipto por cokrigagem, krigagem e regressão. Cerne 2015; 21(2): 243-250. http://dx.doi.or g/10.1590/01047760201521021532.

Mello JM, Oliveira MS, Batista JLF, Justiniano PR Jr, Kanegae H Jr. Uso do estimador geoestatístico para 
predição volumétrica por talhão. Floresta 2006; 36(2): 251-260. http://dx.doi.org/10.5380/rf.v36i2.6454.

Mello JM. Geoestatística aplicada ao inventário florestal [tese]. Piracicaba: Escola Superior de Agricultura Luiz de Queiroz, Universidade de São Paulo; 2004.

Montebeller CA, Ceddia MB, Carvalho DF, Vieira SR, Franco EN. Variabilidade espacial do potencial erosivo das chuvas no estado do Rio de Janeiro. Engenharia Agrícola 2007; 27(2): 426-435. http://dx.doi.org/10.1590/ S0100-69162007000300011.

Rocha TB, Cabacinha CD, Almeida R, Paula, A, Santos, RC. Avaliação de métodos de estimativa de volume para um povoamento de Eucalyptus urophylla s. t. Blake no planalto da Conquista- BA. Enciclopédia Biosfera 2010; 6(10): 1-13.
Santana RA. Avaliação de técinicas geoestatísticas no inventário de povoamentos de Tectonas gradis L.f. [dissertação]. Viçosa: Estatística Aplicada e Biometria, Universidade Federal de Viçosa; 2011.

Thaines F, Braz EM, Mattos PP, Thaines AAR. Equação para estimativa de volume de madeira para a região da bacia do rio Ituxi, Lábrea - AM. Pesquisa Florestal Brasileira 2010; 30(64): 283-289. http://dx.doi.org/10.4336/2010. pfb.30.64.283.

Vibrans AC, Sevgnani L, Lingner DV, Gasper AL, Sabbagh S. Inventário florístico florestal de Santa Catarina (IFFSC): aspectos metodológicos e operacionais. Pesquisa Florestal Brasileira 2010; 30(64): 291-302. http://dx.doi. org/10.4336/2010.pfb.30.64.291. 